\title{
Die Schwerpunktbereiche: eine Chance für die Fakultäten
}

\author{
Peter Hommelhoff
}

In der großen Reform der Juristenausbildung von $2002^{1}$ waren die Schwerpunktbereiche einer der vier Eckpfeiler neben der Anwaltsorientierung des Jurastudiums, seiner Europäisierung und Internationalisierung sowie den Schlüsselqualifikationen. ${ }^{2}$ Rechtspolitisch sind diese Schwerpunktbereiche jüngst ins Gerede geraten: 2012 rät der Wissenschaftsrat dringend ihre Umstrukturierung an $^{3}$ und im Anschluss an ihn plädieren manche gar dafür, die Schwerpunktbereiche, da schwerlich reformierbar, gänzlich abzuschaffen. ${ }^{4}$ Daher scheint es gerade jetzt angezeigt, sich mit ihnen noch einmal intensiver zu befassen. Das soll hier in acht Abschnitten geschehen:

\section{A. Worauf zielen die Schwerpunktbereiche $a b$, und welche Überlegungen lagen ihrer Einführung zugrunde?}

Der Bundesgesetzgeber verfolgte mit den Schwerpunktbereichen nicht weniger als sechs Ziele: ${ }^{5}$

1. eine frühzeitige Berufsorientierung der Studierenden schon im Rahmen ihres Universitätsstudiums;

2. über sie (wie schon über die vorherigen Wahlfächer) eine gewisse Ergänzung des Pflichtfachstudiums;

3. eine Vertiefung der mit den Schwerpunktbereichen zusammenhängenden Pflichtfächer;

4. die Vermittlung (europäischer und) internationaler Bezüge des Lehr- und Lernstoffs;

5. die Vermittlung seiner interdisziplinären Bezüge sowie schließlich

6. in den Jurafakultäten deren Schwerpunktsetzung und Profilierung.

Dabei ließ sich der Bundesgesetzgeber für die Einführung der Schwerpunktbereiche im Wesentlichen von drei Überlegungen leiten: ${ }^{6}$

* Der Autor ist em. Ordinarius für Bürgerliches Recht, Handels- und Wirtschaftsrecht, Rechtsvergleichung und ehemaliger Rektor der Ruprecht-Karls-Universität Heidelberg. Der Beitrag ist eine leicht überarbeitete Fassung des Impulsbeitrags, den der Verfasser während der 11. Soldan-Tagung „Das Jura-Studium auf dem Prüfstand“ am 14. Juni 2013 in der Universität Heidelberg erstattet hat.

1 BGBl I 2002, S. 2592-2594; dazu Burgi, in: NJW 2003, S. 2804 ff.; Gilles/Fischer, in: NJW 2003, S. 707 ff.; Hommelhoff/Teichmann, in: JuS 2002, S. 839 ff.

2 Siehe die Beschlussempfehlung und den Bericht des Bundestagsrechtsausschusses (BT-Drs. 14/8629 v. 20.3.2002), Allgemeines S. 11.

3 Wissenschaftsrat, , S. 59.

4 Wolf, in: ZRP 2013, S. 20 (23); s. aber auch die Interview-Äußerungen von Mager/Piekenbrock, in: Körner, „Die Schwerpunktbereiche gehören abgeschafft“, http://www.lto.de/recht/studium-referendariat/s/schwerpunktbereich-abschaffung-heidelberg/ (15.7.2013): „Wir streben die Abschaffung der Schwerpunktbereiche an".

5 Dazu s. Bundestagsrechtsausschuss zu $\ 5$ a II 4 DRiG n.F. (BT-Drs. 14/8629, S. 12).

6 Bundestagsrechtsausschuss zu $\ 5$ a II 4 DRiG n.F. (BT-Drs. 14/8629, S. 12). 
1. Die Wissenschaftlichkeit des Jurastudiums solle zurückgewonnen werden - (also: auch eine Kampfansage an die Ausbildung durch außeruniversitäre Repetitoren).

2. Die Stellung der Fakultäten innerhalb der Juristenausbildung solle gestärkt werden.

3. Jene Rechtsmaterien, die nach der Wahl des individuellen Studierenden für seine spätere Berufsausübung bedeutsam sind, sollten primär universitär-wissenschaftlich vermittelt werden - also nicht erst später in kurzatmigen Zusatzausbildungen außerhalb der Universität durch professionelle Anbieter oder in Fachanwaltskursen.

\section{B. Welche Mängel glaubt man, den Schwerpunktbereichen heute attestieren zu müssen?}

Mustert man die Einwände, die zehn Jahre nach ihrer Einführung gegen die Schwerpunktbereiche erhoben werden, ${ }^{7}$ durch, so lassen sie sich auf sechs fokussieren:

1. Über den akademischen Lehrstoff werde zu viel Breite und zu wenig Tiefe vermittelt, es werde lediglich additiv Detailwissen angehäuft;

2. daraus rühre eine zu geringe Wissenschaftlichkeit des Jurastudiums in den Schwerpunktbereichen her

3. und trotzdem würden die Studierenden stofflich überlastet.

4. Daneben würden sie zu früh und zu hoch auf bloßen Nebengebieten zu Lasten des Grundlagenwissens spezialisiert;

5. die Systemzusammenhänge innerhalb der Rechtsgebiete und darüber hinaus würden in zu geringem Maße durchdrungen;

6. vor allem herrsche eine verbreitet beängstigende Noteninflation.

Vor einer Auseinandersetzung mit diesen Einwänden sollte man das dafür verfügbare Tatsachenmaterial in die Diskussion einzuführen versuchen.

\section{Welche Auswirkungen der Ausbildungsreform 2002 haben die Justizminister der Länder mit Blick auf die Schwerpunktbereiche festgestellt?}

Aus ihren Feststellungen ${ }^{8}$ sind vier Befunde bemerkenswert:

1. Nach ihren eigenen Angaben haben die Studierenden ihre jeweiligen Schwerpunktbereiche zu 93\% aus persönlichem Interesse gewählt, zu 63\% im Hinblick auf ihre Berufsvorstellungen und lediglich zu $17 \%$ aus prüfungstaktischen Gründen. Das deckt sich ganz weitgehend mit den Einschätzungen der Juristischen

7 Wissenschaftsrat, Perspektiven der Rechtswissenschaft, S. 59; Wolf, in: ZPR 2013, S. 20 (23); Mager/ Piekenbrock, in: Körner, „Die Schwerpunktbereiche gehören abgeschafft“, http://www.lto.de/recht/ studium-referendariat/s/schwerpunktbereich-abschaffung-heidelberg/ (15.7.2013); s. auch schon Windel, in: Jura 2003, S. 79: Scheinspezialisierung und Verzettelung.

8 Vgl. Justizministerkonferenz, Bericht über die Auswirkungen des Gesetzes zur Reform der Juristenausbildung, passim. 
Fakultäten; ${ }^{9}$ abweichend von den studentischen Angaben jedoch sehen die Fakultäten die Bereichswahl zu über $70 \%$ als prüfungstaktisch motiviert an.

In summa halten die Justizminister deshalb fest: Das Reformziel des Gesetzgebers von 2002 sei erreicht worden; ${ }^{10}$ die Studierenden könnten ihren Schwerpunkt nach den eigenen Neigungen und Berufswünschen setzen und täten dies auch.

2. Der außeruniversitäre Repetitor spielt für die Prüfung im Schwerpunktbereich eine allenfalls marginale Rolle; dagegen nehmen mehr als 80\% der Studierenden für die Pflichtfachprüfungen die Hilfe eines Repetitors in Anspruch. ${ }^{11}$

Daraus folgt: In den Schwerpunktbereichen haben die Fakultäten die „Lufthoheit" für die Juristenausbildung zurückerobert.

3. Ermutigend konsequent ist die von den Justizministern berichtete Einschätzung der Fakultäten: Zwei Drittel von ihnen sieht die Wissenschaftlichkeit des Jurastudiums durch die Prüfung in den Schwerpunktbereichen als gesteigert an und führt dies im Wesentlichen auf die Seminar- bzw. Studienarbeit zurück. ${ }^{12}$

4. Zum eigenständigen Profil der Fakultäten: Auffällige fast $90 \%$ von ihnen sind der Ansicht, mit dem Zuschnitt ihrer Schwerpunktbereiche ein besonderes Profil entwickelt zu haben, das sich von dem anderer Fakultäten markant unterscheide. ${ }^{13}$ Indes - diese Selbsteinschätzung bedarf wohl doch noch einer objektiven Gegenlese anhand der jeweiligen Strukturpläne.

Aber wie auch immer: Mit Blick auf die Ziele der Ausbildungsreform 2002 sind all dies erfreuliche Feststellungen der für die Juristenausbildung mitverantwortlichen Justizminister. Hinzu kommt ihre anerkennende Feststellung, die internationale (und wie zu ergänzen ist: die europäische) Ausrichtung des Lehrangebots in den Jurafakultäten könne als beeindruckend bezeichnet werden. ${ }^{14}$ Diese ministeriellen Feststellungen erlauben einige Kommentare.

\section{Worin liegen die Vorteile der Schwerpunktbereiche für Studierende und Fakultäten?}

Die neuen Schwerpunktbereiche haben den Studierenden, aber auch den Juristischen Fakultäten schon jetzt reichen Gewinn gebracht:

9 Justizministerkonferenz, Bericht über die Auswirkungen des Gesetzes zur Reform der Juristenausbildung, S. 14/56.

10 Justizministerkonferenz, Auswirkungen des Gesetzes zur Reform der Juristenausbildung, S. 17.

11 Justizministerkonferenz, Auswirkungen des Gesetzes zur Reform der Juristenausbildung, S. 16.

12 Justizministerkonferenz, Auswirkungen des Gesetzes zur Reform der Juristenausbildung, S. 60 f.; a .A. Piekenbrock, in: Körner, Die Schwerpunktbereiche gehören abgeschafft, http://www.lto.de/recht/studium-referendariat/s/schwerpunktbereich-abschaffung-heidelberg/ (15.7.2013): die Schwerpunktbereichsprüfung sei so bürokratisiert, dass die Wissenschaftlichkeit des Studiums wieder verloren gehe. Das kann ich aus eigener Erfahrung nicht bestätigen.

13 Justizministerkonferenz, Auswirkungen des Gesetzes zur Reform der Juristenausbildung, S. 56.

14 Justizministerkonferenz, Auswirkungen des Gesetzes zur Reform der Juristenausbildung, S. 63. 
- Die Studierenden erlernen jetzt wieder ein betont wissenschaftliches Arbeiten und müssen dies auch mit Blick auf die Prüfung ihrer Leistungen durch Wissenschaftler.

- Für ein solch betont wissenschaftliches Arbeiten sind die Studierenden besonders aufgeschlossen, weil sie ihren eigenen Schwerpunktbereich zu über $90 \%$ aus persönlichem Interesse gewählt haben.

- Für die Dozenten bietet dies zugleich die verheißungsvolle Chance, „ihre“ Studierenden für ihr Fach zu entflammen und zu begeistern.

- Darüber hinaus bieten die Schwerpunktbereiche dem Dozenten hier und da die Chance, seine aktuelle Forschungen in den akademischen Unterricht einzubringen und die Studierenden an ihnen teilhaben zu lassen.

- Eine so betont wissenschaftsbasierte Lehre erlaubt es den Jurafakultäten und ihren Universitäten, sich gegenüber den Fachhochschulen und deren Lehrangeboten abzugrenzen; diese sollen vornehmlich der Wissensvermittlung und der Bewältigung von Routinearbeiten dienen. In den Universitätsfakultäten dagegen ist die betont wissenschaftsbasierte Lehre von zentraler Bedeutung für deren exklusives Promotionsrecht - auch gegenüber den außeruniversitären Forschungseinrichtungen.

Insgesamt gilt: Vor allem die Schwerpunktbereiche verleihen dem Jurastudium als Professionsstudium ${ }^{15}$ (mit den Pflichtfächern in seinem Zentrum und als Fundament) jene Wissenschaftlichkeit in Lehre und Prüfung, die unverzichtbar notwendig ist, um das universitäre Jurastudium in seiner spezifischen Eigenständigkeit abzugrenzen gegenüber Fachhochschul-Studiengängen und Fortbildungskursen in der Berufsphase, aber auch, um dem überkommenen Jurastudium Widerstandskraft gegen Bachelor/ Master-Bestrebungen für die Juristenausbildung zu verleihen. ${ }^{16}$

Vor diesem Hintergrund kann eine Rückkehr zu den alten Wahlfachgruppen nicht ernsthaft im rechtspolitischen Raum diskutiert werden, aber erst recht nicht in den Universitäten. Schon heute (insbesondere nach dem zweifachen Durchlauf des Wettbewerbs in der Exzellenzinitiative mit geringen Erfolgen der Rechtswissenschaft) bedarf das Ansehen der juristischen Fakultäten im Kreis der universitären Schwesterfakultäten steter und intensiver Pflege. Allerdings - gerade wegen der Wissenschaftlichkeit des Jurastudiums muss man sich mit den behaupteten Mängeln der Schwerpunktbereiche näher befassen.

\section{E. Welche Gegenargumente gegen die Schwerpunktbereiche wiegen zu leicht?}

Über einige der Einwände sollte man alsbald zur Tagesordnung übergehen:

1. Die Spezialisierung in angeblichen Nebengebieten; dazu aus dem (zugegeben ganz subjektiven) Blickwinkel eines Wirtschaftsrechtlers: Unternehmens-, Bilanz- und Steuerrecht sind schon je für sich genommen von viel zu großem praktisch-pro-

15 S. Wissenschaftsrat, Perspektiven der Rechtswissenschaft, S. 7.

16 S. dazu die Beiträge in Baldus/Finkenauer/Rüfner (Hrsg.), Bologna und das Rechtsstudium, 2011. 
fessionellen Gewicht (z.B. weit mehr als 1 Mio. Gesellschaften mbH), ${ }^{17}$ viel zu fundamental für unsere Rechts-, Wirtschafts- und Sozialordnung, als dass sie in den Universitäten in Forschung und Lehre ausgeblendet werden dürften. Ohne ihre Einbeziehung in den akademischen Unterricht wären jene Studierenden, die in diesem Bereich später ihren Unterhalt verdienen wollen, für ein bald vierzigjähriges Berufsleben mitsamt all seinen heute überhaupt noch nicht absehbaren Herausforderungen und Veränderungen nicht zukunftsfähig genug aus- und vorgebildet. Juristischer Generalismus allein schafft die Voraussetzungen hierfür nicht. - Andere mögen Beispiele aus ihren Schwerpunktbereichen beisteuern.

2. Die angeblich in den Schwerpunktbereichen fehlende Systemverknüpfung: In der Tat zählen Systemverknüpfungen zu den Fundamenten wissenschaftlichen Arbeitens; im Zivilrecht allerdings nicht allein und auch nicht so sehr zwischen Gesellschafts- und Bilanzrecht auf der einen Seite und BGB auf der anderen. ${ }^{18}$ Verknüpfungen finden sich vielmehr

- zwischen Gesellschafts- und Bilanzrecht als Grundlage für Kapital-, Gläubigerund Minderheitenschutz ${ }^{19}$,

- zwischen Gesellschaftsrecht und den Grundprinzipien der Privatautonomie: die gesellschaftsrechtlichen Freiheiten ${ }^{20}$ und Selbstgestaltungsmacht sowie Eigenverantwortlichkeit versus Staatsfürsorge ${ }^{21}$,

- zwischen Unternehmens- und Verfassungsrecht: staatlich gesetztes Recht versus Selbstregulierung der Wirtschaft, ihrer Akteure und Unternehmen ${ }^{22}$.

Fazit (zumindest für das Unternehmens- und Wirtschaftsrecht): von fehlender Systemverknüpfung kann nicht ernsthaft die Rede sein.

3. Die perhorreszierte Noteninflation: richtig, nach Erhebung der Landesjustizminister sind die Ergebnisse in den Schwerpunktbereichsprüfungen in 73\% der Fälle besser als in den Pflichtfachprüfungen. ${ }^{23}$ Aber - juristische Befähigung erschöpft sich nicht in der Fall-Lösung (ohne deren ebenfalls wissenschaftlichen Gehalt in Abrede stellen zu wollen); ganz offenbar macht wissenschaftliches Arbeiten den Studierenden Freude und weckt ihre Kreativität. Das schlägt sich dann im Leistungsergebnis nieder und findet seine Erklärung nicht etwa im Wettbewerb der Professoren um eine möglichst große Zahl von Studierenden in ihrem Schwerpunktbereich. ${ }^{24}$ Warum sollten prüfende Professoren in der Universitätsprüfung

17 Kornblum, in: GmbHR 2013, S. 693 (703) hat zum 1.1.2013 in Deutschland insgesamt 1.098.222 Gesellschaften mit beschränkter Haftung ermittelt.

18 Dazu aber jüngst etwa der Hinweisbeschluss des OLG Stuttgart vom 20.11.2012 - 1411 39/12, NZG 2013, 618 - Leitsätze.

19 Näher Hommelhoff, in: GS Schindhelm, S. 365 ff.

20 Rittner, Die werdende juristische Person, S. $248 \mathrm{ff}$.

21 Lutter, in: GmbHR 1990, S. 337 (339).

22 Dazu u.a. näher Bachmann, Private Ordnungen, passim.

23 Justizministerkonferenz, Auswirkungen des Gesetzes zur Reform der Juristenausbildung, S. 15; s. hierzu aber auch die Beobachtung von Mager, in: Baldus (Hrsg.), S. 247.

24 Wie in der Diskussion ein Juraprofessor behauptete. 
andere Maßstäbe anlegen als in der Staatsprüfung? Und wo das (nach meiner Beobachtung allenfalls äußerst ausnahmsweise) tatsächlich so sein sollte, ist eine solche Fehlentwicklung collegialiter unter Einschaltung des Dekans oder Studiendekans zu bereinigen. Jedenfalls sprechen solche möglichen Ausreißer im Einzelfall mitnichten gegen die Schwerpunktbereiche als solche.

\section{F. Wo liegen die wirklichen Schwachstellen der Schwerpunktbereiche?}

Sie lassen sich unter sechs Stichworten zusammenfassen:

1. die weithin unzureichende Einbeziehung der Grundlagen in die Schwerpunktbereiche (im Wirtschaftsrecht z.B. die nahezu überall fehlende Wirtschaftsrechtsgeschichte der Neuzeit) ${ }^{25}$;

2. die weit verbreitet zu frühe Positionierung der Schwerpunktbereiche im Curriculum mit der Folge, dass die Studierenden mit zu geringem juristischem Vorund Allgemeinwissen und noch zu geringer juristischer Befähigung bereits mit den häufig besonders herausfordernden Themen der Schwerpunktbereiche befasst werden;

3. der viel zu schwache Einsatz wissenschaftsorientierter Lehrtypen (Seminare, Kolloquien etc.);

4. die überbordende Prüfungsbelastung des Lehrpersonals ${ }^{26}$;

5. die immer noch ganz unzureichende Betreuungsrelation, d.h. der Curricularnormwert 2,2, der in manchem Bundesland über den jüngeren Zuwachs an Studierenden schon mehr oder minder weit wieder unter 2,0 abgesenkt worden ist;

6. die verfehlt nahezu ausschließlich auf den Staatsteil des Ersten Examens ausgerichtete Einschätzung der Arbeitgeber - auch und vor allem in der Anwaltschaft $^{27}$.

\section{G. Wie sollten die Schwerpunktbereiche aufgefrischt werden?}

Diese Schwachstellen müssen beseitigt werden; daraus ergeben sich eine Reihe von Konsequenzen:

1. Der Umfang der Schwerpunktbereiche sollte in sämtlichen Bundesländern von 16 auf 24 Semesterwochenstunden erweitert werden. ${ }^{28}$ Das ist für die Stoffvermittlung einerseits und die eigenständige Stoffdurchdringung der Studierenden andererseits unumgänglich.

25 Zum Wirtschaftsrecht als Grundlagenfach Rittner/Dreher, Europäisches und deutsches Wirtschaftsrecht, S. $1 \mathrm{ff}$.

26 Auf sie haben zu recht schon Horz/Katzenstein, in: VBlBW 2006, S, 1 (3 f.) hingewiesen; s. aber bereits Hommelhoff/Teichmann, in: JuS 2002, S. 839 (841, 843).

27 Das frustriert auch und vor allem die Fakultäten in ihrem enormen Aufwand in den Schwerpunktbereichsprüfungen, s. Mager/Piekenbrock, in: Körner, „Die Schwerpunktbereiche gehören abgeschafft“, http://www.lto.de/recht/studium-referendariat/s/schwerpunktbereich-abschaffung-heidelberg/ (15.7.2013).

28 Dazu schon Hommelhoff, in: FS Otte, S. 123 (127). 
2. Im Gegenzug müssen der Pflichtstoff und der zeitliche Aufwand für ihn um 8 Semesterwochenstunden im Gesamtcurriculum reduziert werden. ${ }^{29}$ Das nimmt den jungen Juristen gewiss nicht die spätere Befähigung eines generalistischen Volljuristen.

3. Die Vielfalt der Typen akademischer Lehrveranstaltungen (vor allem in Kleingruppen) muss angereichert werden.

4. In diese Lehrveranstaltungen sind verstärkt die Grundlagenfächer mit einzubeziehen und dort, wo wirklich Gewinn zu erwarten ist, sind auch interdisziplinäre Bezüge herzustellen. Europäisches und Internationales hingegen scheint schon jetzt ausreichend berücksichtigt.

5. Die Prüfungsbelastungen der einzelnen Dozenten müssen drastisch reduziert wer$\operatorname{den}^{30}$.

6. Für die Schwerpunktbereiche und die Prüfungen in ihnen müssen die Qualitätskontrollen ${ }^{31}$ spürbar verbessert werden.

7. Die Betreuungsrelation bedarf durchgreifender, effektiver und nachhaltiger Steigerung.

8. Die Juristischen Fakultäten müssen ganz erheblich besser finanziert werden; d.h.: der finanzielle Aufwand/Student ist wesentlich anzuheben. ${ }^{32}$ Das Jurastudium ist kein billiges und immer stärker beschickbares Aktionsfeld zur Bewältigung von Studentenbergen.

9. Um auch dem Universitätsteil des Ersten Examens das notwendige Gewicht zu verleihen, sollten die staatlichen Arbeitgeber damit beginnen, sich allein am Gesamtergebnis des Ersten Examens zu orientieren und nicht ganz vordringlich am Ergebnis des Staatsteils. Die Fakultäten und ihre Professoren sollten ihre Netzwerke in die Praxis nutzen, um für die Bedeutung und den Wert der Schwerpunktbereiche und deren Prüfung zu werben. Dagegen würde der unaufdeckbare Einheitsausweis des Ergebnisses im Ersten Examen dem Transparenzgebot widersprechen. ${ }^{33}$

10. Indes - vielleicht wird man diesem Problem noch radikaler zu Leibe rücken müssen: In der Ersten Prüfung darf der Universitätsteil nicht zum bloßen Anhängsel des Staatsteils verkommen; beiden Teilen muss vielmehr gleiches Gewicht und gleiche Reputation verschafft werden. Deshalb sollte auch der Universitätsteil der Ersten Prüfung von den staatlichen Prüfungsämtern administriert werden. Um die Gleichstellung der Prüfungsteile im praktischen Prüfungsgeschehen durchge-

29 Mit dieser Forderung stehe ich in klarem Gegensatz zu Mager/Piekenbrock, in: Körner, „Die Schwerpunktbereiche gehören abgeschafft", http://www.lto.de/recht/studium-referendariat/s/schwerpunktbereich-abschaffung-heidelberg/ (15.7.2013), die für eine Rückkehr zum Zustand vor der Ausbildungsreform 2002 (mit Ausnahme der Anwaltsorientierung) plädieren. Kritisch zur Erhöhung der Schwerpunkt-Stunden aber auch schon Horz/Katzenstein, in: VBIBW 2006, S. 1 (3).

30 Die völlige Ausblendung der Prüfungslast juristischer Dozenten soll, wie man hört, in eklatantem Gegensatz zur Handhabung in anderen Fächern stehen.

31 Hierauf macht mit Recht Mager, in: Baldus u.a. (Hrsg.), S. 239, 249 aufmerksam.

32 Auch vor dem Hintergrund einer deutlich zu verbessernden Lehr- und Prüfungsqualität in den Schwerpunktbereichen ist die bundesweite Abschaffung von Studienbeiträgen zu bedauern.

33 In diesem Punkt hat mich ein Diskussionsbeitrag überzeugt. 
hend zu gewährleisten, sollten über die Professoren als Mitglieder der Prüfungsämter hinaus diese durch eine präsidiale Doppelspitze geführt werden, der ein hauptamtlicher Hochschullehrer angehört. ${ }^{34} \mathrm{Er}$ ist auf der Grundlage seiner Wahl durch sämtliche Hochschullehrer im Amtsbezirk dem Justizminister zur Berufung vorzuschlagen.

\section{H. Wer sollte was tun?}

- Möglichst viel die Fakultäten in Profil schärfender Eigenverantwortlichkeit vor allem bei der Ausgestaltung der Schwerpunktbereichsprüfungen.

- Die Landesgesetzgeber sollten sich der Bemessung des Lehr- und Prüfungsstoffs in Pflichtfächern und Schwerpunktbereichen erneut annehmen und die Justizprüfungsämter universitätsorientiert reorganisieren.

- Die Landesjustiz- und -wissenschaftsminister sollten bei ihren Finanzministern mit größtem Nachdruck für eine wettbewerbsfähig finanzierte Juristenausbildung streiten. ${ }^{35} \mathrm{Ob}$ sich zusätzlich Bundesmittel erschließen lassen, sollte mit allem Ernst geprüft werden. ${ }^{36}$

- Der Bundesgesetzgeber sollte prüfen lassen, wo und warum seine mit der Ausbildungsreform 2002 angestrebten Ziele noch nicht überall erreicht sind.

Das alles sollten die Juristen selbst in die Hand nehmen, bevor ihnen andere, etwa im Wissenschaftsrat, vorgeben, wie sie zu forschen und zu lehren haben. Die Rückkehr zu den Zeiten vor der Ausbildungswende ist keine vernünftige Option; vor allem sollte sie dies nicht in jenen Fakultäten sein, die die Reform 2002 wesentlich mitgestaltet haben.

\section{Literaturverzeichnis}

Ausschuss der Konferenz der Justizministerinnen und Justizminister zur Koordinierung der Juristenausbildung: Bericht über die Auswirkungen des Gesetzes zur Reform der Juristenausbildung - Fortsetzung der Evaluation für den Zeitraum Januar 2007 bis Oktober 2010, abrufbar unter http://www.justiz.nrw.de/ $\mathrm{JM} /$ justizpolitik/schwerpunkte/juristenausbildung/evaluation/bericht2011.pdf (15.7.2013).

Bachmann, Gregor, Private Ordnung - Grundlagen ziviler Regelsetzung, Tübingen 2006.

34 Für diese Anregung danke ich Herrn Professor Thomas Pfeiffer, Heidelberg.

35 „Fehlt für eine Reform das Personal, muss man sie zurücknehmen“ (so Piekenbrock, in: Körner, „Die Schwerpunktbereiche gehören abgeschafft", http://www.lto.de/recht/studium-referendariat/s/schwerpunktbereich-abschaffung-heidelberg/ (15.7.2013)), ist die falsche Strategie.

36 In diesem Zusammenhang gewinnt die neueste Verlautbarung des Wissenschaftsrats vom 12. Juli 2013 „Perspektiven des deutschen Wissenschaftssystems“ mit Hintergrundinformation vom 15. Juli 2013 „Zukunftspakt für das Wissenschaftssystem“ herausragende Bedeutung. Rückt der Rat doch in seinem Empfehlungen an die Bundesregierung und an die Regierungen der Länder zur Zukunft des Wissenschaftssystems für die Hochschulen die zu verbessernde Qualität von Studium und Lehre an die erste Stelle (Hintergrundinformation, S. 1), verweist auf die Notwendigkeit, die Betreuungssituation generell sowohl quantitativ als auch qualitativ zu verbessern (Verlautbarung, S. 33 f.) und erwähnt in diesem Zusammenhang die sehr ungünstigen Betreuungsrelationen speziell in den Wirtschafts- und Rechtswissenschaften (Verlautbarung, S. 34). Konsequent müssen die zusätzlich von den Ländern und vom Bund eingeforderten Mittel auch und vor allem in verbesserte Lehr- und Studienbedingungen investiert werden. Hierzu zählen auch die Kosten für die Durchführung von qualitätsvollen Prüfungen. 
Baldus, Christian/Finkenauer, Thomas/Rüfner, Thomas (Hrsg.), Bologna und das Rechtsstudium - Fortschritte und Rückschritte der europäischen Juristenausbildung Tübingen 2011.

Burgi, Martin, Die glückende Reform: Zur neuen Juristenausbildung an den Universitäten, in: NJW 2003, S. 2804-2805.

Gilles, Peter/Fischer, Nikolaj, Juristenausbildung 2003 - Anmerkungen zur neuesten Reform, in: NJW 2003, S. 707-711.

Hommelhoff, Peter/Teichmann, Christoph, Das Jurastudium nach der Ausbildungsreform, in: JuS 2003, S. 839-844.

Hommelhoff, Peter, Zur Umsetzung der Juristenausbildungsreform 2003 auf Länderebene in: Baumann/ Dickhuth-Harrach/Marotzke (Hrsg.), Gesetz - Recht - Rechtsgeschichte, Festschrift für Gerhard Otte, München 2005, S. 123-133.

Hommelhoff, Peter, Gesellschaftsrechtliche Implikationen des BilMoG, in: von Bar (Hrsg.), Recht und Wirtschaft, Gedächtnisschrift für Malte Schindhelm, Köln 2009, S. 365-376.

Horz, Cornelia/Katzenstein, Matthias, Zur Umsetzung der Reform der Juristenausbildung in Baden-Württemberg, in: VBlBW 2006, S. 1-8.

Kornblum, Udo, Bundesweite Rechtstatsachen zum Unternehmens- und Gesellschaftsrecht (Stand 1.1.2013), in: GmbHR 2013, S. 693-703.

Lutter, Marcus, Das System der Kapitalgesellschaften, in: GmbHR 1990, S. 377-379.

Mager, Ute, Die Ausbildungsreform von 2002 - Ziele, Inhalte, Erfahrungen und Folgerungen für weitere Reformen, in: Baldus/Finkenauer/Rüfner, Bologna und das Rechtsstudium - Fortschritte und Rückschritte der europäischen Juristenausbildung, Tübingen 2011, S. 239-252.

Rittner, Fritz, Die werdende juristische Person - Untersuchungen zum Gesellschafts- und Unternehmensrecht, Tübingen 1973.

Rittner, Fritz/Dreher, Meinhard, Europäisches und deutsches Wirtschaftsrecht: Eine systematische Darstellung, 3. Aufl., Heidelberg 2008.

Wissenschaftsrat (Hrsg.), Perspektiven der Rechtswissenschaft in Deutschland. Situation, Analysen, Empfehlungen, Drs. 2558-12, Hamburg 2012.

Wolf, Christian, Perspektiven der Rechtswissenschaft und der Juristenausbildung - Kritische Anmerkungen zu den Empfehlungen des Wissenschaftsrates, in: ZRP 2013, S. 20-23. 